\title{
MAXIMIZING THE AMOUNT OF PICKED FRUIT REGARDLESS OF SPECIES AND VARYING CHARACTERISTICS BY IMPLEMENTING PRINCIPLES OF HUMAN - ROBOT INTERACTION IN GREENHOUSES - MODEL MULTIHBOT
}

\author{
Sara Gračić 333
}

https://doi.org/10.31410/itema.2018.974

\begin{abstract}
The expectation of increasing yields to meet the growing food demand of the increasing population resulted in the new agricultural trend of producing robust machinery combined with precise farm traffic as well as a high price to pay. Constant high pressure on the soil and repeatedly traversing exactly the same route results in concentrated soil compaction damage and machine failures decrease productivity and income. If possible, investments in new machines do not guarantee the increase of goods in the same amount. Farmers work in harsh, even hazardous conditions (pesticides, fungicides and herbicides for plant protection), which have negative impact on their health. On the other hand, science has advanced in the field of robotics, so possibilities and effects of implementing these achievements in agriculture are being examined. Agricultural robots (AGROBOTS) are an extension of a human hand with the goal of replacing him/her in performing difficult tasks and/or in hazardous environments and/or with a variable climate. They can traverse unstructured, unpredictable terrains, while being exposed to the environment. It is expected of them to perform very complex tasks e.g. picking different types, sizes, shapes and colors of fruits, in a highly variable environment where illumination is unequal, locations are random and undefined and climate related factors can drastically worsen working conditions (mud, strong winds, dust, different light settings depending on the position of the sun and the clouds), which will affect their performance. Therefore, it is important to understand the principles of Human-Robot Interaction (HRI), which gives insight into how people and machines can collaborate to achieve desired results. Based on literature review of scientific papers from Google Scholar and Mendeley, the author has identified two problems related to agrobots, which will be in focus of this research. First problem is related to harvesting fruits in real surroundings, because robots are not always able to distinguish a leaf from a fruit. Second problem is that these robots are limited to picking only one type of fruit. To solve these problems, MultiHBot, a robotic model based on HRI was designed. Human controls the picking process via Wi-Fi console through robotic vision, arms and scissors. When a human spots a fruit, he/she instructs the robot to "harvest" it. This ensures that all fruits will be picked, thus maximizing performance which is measured in quantity of picked fruit and revenue. This also means that MultiHBot can be used for harvesting every culture, thus eliminating the need for having different robots for different cultures, with an obvious benefit of cost cutting. MultiHBot could also be used for spraying seeds, if necessary. The robot's movement along the floor rail is automated and positioning is based on sensors. Combining human intelligence for performing complex part of procedures with robotic automation of simple parts will eliminate physical presence in the field and ensure successful completion of the whole process, increase in productivity and income.
\end{abstract}

Keywords: Agrobots, Human-Robot interaction, process

\footnotetext{
${ }^{333}$ University of Novi Sad, Faculty of Economics, Segedinski put 9-11, Serbia
} 


\section{INTRIGUING FACTS ABOUT (MODERN) AGRICULTURAL PRODUCTION}

It is expected of agriculture to increase yields to meet ever-growing food demand of the increasing world population, which essentially means more intense crop and cattle cultivation to produce more food [1]. This has resulted in a trend of manufacturing robust agricultural machinery combined with precise farm traffic [2] and a high price to pay. Constant high pressure on the soil, due to complexity, size and weight of the equipment and repeatedly traversing exactly the same route results in concentrated soil compaction damage and machine failures decrease productivity and income [2].

Assuming investing in the state-of-the-art agricultural machinery is possible (which depends of farmers' financial strength), increase in production in the same ratio is not guaranteed. Production volume is determined by the surface of cultivable area which cannot be increased; soil fertility; matching different cultures with adequate type of soil; specific area's climate; irrigation systems etc. Serbia is faced with migration to cities, brain drain and white plague, which are erasing villages, thus leaving hectares of fertile land uncultivated. Floods are causing problems due to inadequate protection systems.

Farmers work in harsh, even hazardous conditions (pesticides, fungicides and herbicides for plant protection), which have negative impact on their health. On the other hand, science has advanced in the field of robotics, so possibilities and effects of implementing these achievements in agriculture are being examined.

\section{ROBOTICS RESPONDS TO SPECIFIC AGRICULTURE CONDITIONS}

Due to unfavorable/specific conditions in agriculture [3], robots are being applied as a perspective alternative to decreased number of farmers, which is considered a mayor bottleneck in agricultural production [4], [5]. AGROBOTS are an extension of a human hand with the goal of replacing him/her in performing difficult tasks and/or in hazardous environments [6] and/or with variable climate.

They can traverse unstructured, unpredictable terrains [7], while being exposed to the elements [6]. It is expected of them to perform very complex tasks e.g. picking different types, sizes, shapes and colors of fruits, in a highly variable environment where illumination is unequal, locations are random and undefined and climate related factors can drastically worsen working conditions (mud, strong winds, dust, different light settings depending on the position of the sun and the clouds) [7], which will have effect on the quality of their performance [6]. So, it is important to understand the principles of Human-Robot Interaction, which gives insight into how people and machines can collaborate to achieve desired results.

\section{PRINCIPLES OF HUMAN-ROBOT INTERACTION (HRI)}

HRI is committed to understanding, designing and evaluating robots for use by humans or their collaboration [8], it includes knowledge in robotics, human factors, cognitive science, natural language, psychology and human-computer interaction to understand their relationship [7].

HRI can be: remote - human and robot are separated spatially or temporally and proximatewhen they are collocated [9]. Robots can be completely automated and with their further development, it will be expected of them to perform more complex tasks [7]. By including a 
human, robots' performances improve, which can lead to cost cutting and economic feasibility of a project - a limiting factor for commercialization of AGROBOTS [10].

Instead of autonomous robots, use of teleoperations [11] based on HRI [8] is proposed. In HR relationship, this is a human's understanding of location, activity, status, environment of the robot and the level of certainty related to these data. In RH relationship, this is the knowledge a robot has of human's commands and restrictions that may require changing course of action or command noncompliance.

Awareness of the location means understanding where the robot is currently located or moving towards, where it was, where it still needs to go. Awareness of activities means understanding what the robot is doing and its progresses, whether human intervention is needed and is the robot performing tasks it should. Awareness of status means understanding information about the platform, computer system, cameras, sensors, speed, energy level and status of a task, e.g. the remaining amount of spraying liquid. Surroundings awareness means knowing what is around the robot while performing tasks e.g. farmers, other robots and weather conditions. Overall mission awareness means understanding all of the above stated and deciding on future robot activities.

The advantage is in combining human "know how" and robot's accuracy, repetitiveness and strength to remove farmers from hazardous locations [7], e.g. when spraying plants [12], ease of use and better performance [13]. Still, farmers must be on the alert, and the question, for some, remains: are efficiency, comfort and health worth the cost of such systems [7]? Those should get familiarized with work-related diseases and economics of healthcare systems.

\section{RESEARCH BACKGROUND}

BoniRob (V2) with greater robustness for continuous outdoor use and a reusable platform for multiple agriculture purposes with application modules ("BoniRob-Apps") was introduced in [16]. Penetrometer App integrates a mechanical actuator for measuring soil properties which penetrates to a depth of about $80 \mathrm{~cm}$ and shows constant characteristics and allows replications for a large number of measurements. Phenotyping App is used for plant characterization based on multi-sensor applications, including morphological and spectral characteristics, which will enable single plant management. Precision-Spraying App detects green surfaces and sprinkles only plants with herbicides, thus drastically reducing the amount of pesticide used.

In [14] empirical evidence in favor of human-robot cooperation in target recognition tasks are presented. Detection was increased by $4 \%$ compared to a human and by $14 \%$ compared to a fully autonomous robot and detection time was reduced [15].

The authors in [7] developed a robot for selective vineyard spraying. Traditionally, hand sprayer or tractor carrying sprinklers is used, thus resulting in excessive use of pesticides and endangering a farmer's health. The HR sprinkler enables targeted spraying and decreases pesticides usage, while a farmer is at a safe distance.

In the AURORA project [17], a robust, low-cost robot, capable of autonomous navigation in different types of greenhouses, is proposed. Authors in [18] present a robot that monitors plants' health, dispersion of chemicals, medicines and fertilizers in greenhouses. 
In [19], an autonomous mobile robot controls pests and is in charge of disease prevention in commercial greenhouses. It can independently navigate through the rows, while pesticide spraying system covers plants evenly in defined dosages.

Navigation methods of mobile agricultural robots in greenhouses are discussed in [20]. Many problems are caused by repetitive, dangerous tasks performed by humans, which can be easily avoided by using robots. Autonomous navigation was solved by using intended and pseudoreactive techniques. The first uses a mapping algorithm to create a safe path without obstacles. The second uses feedback from the sensors to move safely through the greenhouse. Both approaches were tested in a real environment, with promising results.

A robot for the recognition and cutting of sweet peppers in greenhouses was introduced in [21]. It has a built-in image processing system with parallel stereo vision. A prototype has been made. Experiments show that the robot's performance depends of the fruit recognition, since it has the ability to choose sweet peppers. The left camera has to recognize a sweet pepper. If it does, it calculates the distance on the left side along the $\mathrm{x}$ and $\mathrm{y}$ axis. The right camera performs the same task. If both cameras detect a fruit, 3D coordinates are determined. The scissors open, harvest the fruit, insert it into a basket, close and the robot continues the search. In the first experiment, all peppers were successfully cut, because there were no leaves. In the second, carried out in realistic conditions, all the peppers were identified, but only those on the left and the centre were cut, while the peppers on the right were not, because the robot did not see them from the leaves.

\section{PROBLEMS IN FOCUS OF THIS RESEARCH}

The experiments in [21] showed that harvesting fruits in realistic conditions is not accurate and the robot is limited to a specific type of fruit. First problem in focus of this research is to ensure that a robot picks all the fruits from a plant in order to maximize the effect, i.e. to harvest all fruits to provide more food and maximize revenue. Second problem in focus is to extend harvesting capabilities to other cultures, thus creating a universal greenhouse robot.

\section{MULTIHBOT}

MultiHBot is a robotic system based on HR interaction principles. It enables human control of the processes of identifying and cutting fruits and it can be used for picking any type of fruit, by using human knowledge and instructions. The robot has a camera for visualization, two robotic arms, with 7 degrees of freedom, for moving leaves and scissors for cutting fruits.

The robot is mounted on floor rail used for circulating through the greenhouse. Human interacts with the robot via console and voice commands. Sensors are set in front of each plant. When a robot registers the signal, it stops, rotates the camera, basket, arms and scissors towards the plant.

The console has two circles, each for maneuvering one robotic arm. A human uses the circles when performing leaf coverage check-up. If the check-up results in a covered fruit, a human has the ability to position robotic arms and direct the scissors to cut the fruit. The robot puts the fruit in the basket.

At the end of each lane, the basket is changed, thus ensuring that the weight of picked fruits does not cause problems in speed or excessive pressure on the floor rail. When the robot is 
passing by the basket to enter the next lane, the basket registers it and automatically goes to the entrance using the same floor rail. When the processes are finished, robot and baskets are returned to their initial starting points.

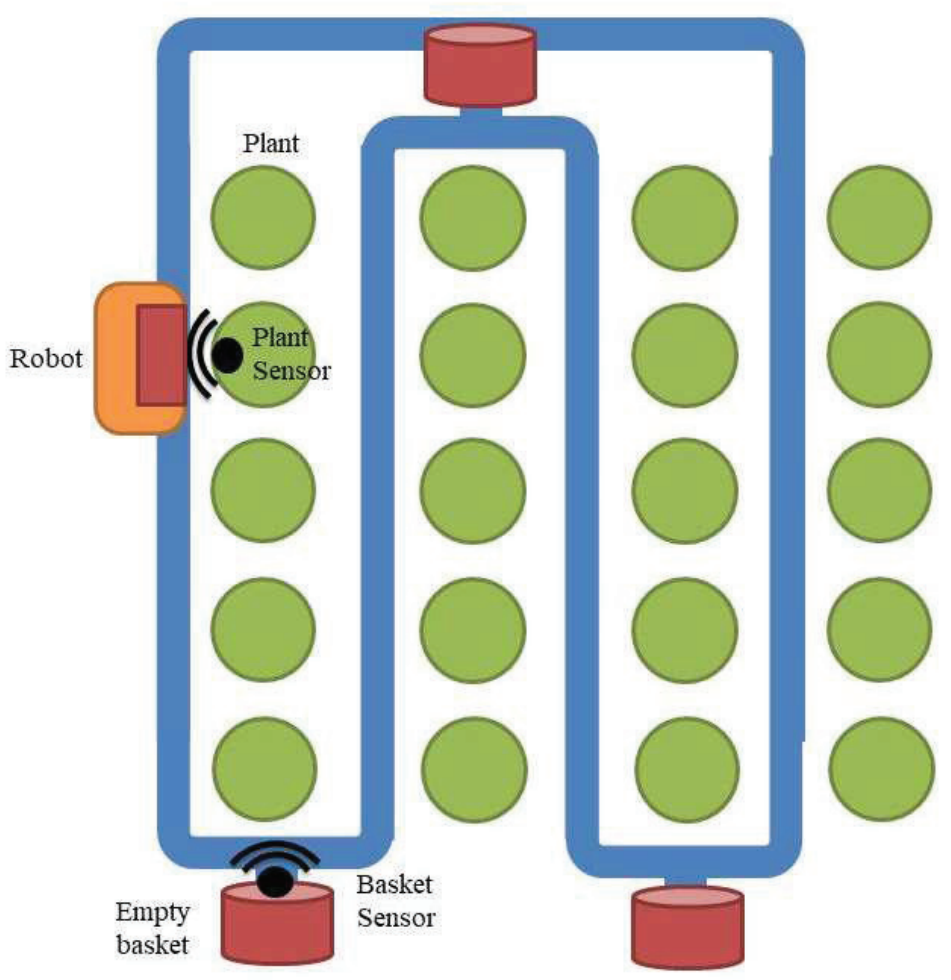

Figure 1: MultiHBot picking fruits in a greenhouse

In this way, the problem of not differentiating a fruit from a leaf is solved, as well as the problem of constructing robots for picking only one type of fruit, because human intelligence distinguishes leaf from fruits, regardless of species. Robotic field work will enable the replacement of human presence in a classical working environment, while ensuring successful execution of tasks in order to increase productivity and consequently, income.

Although the model could also be used for spraying seeds, it will not be used for this purpose, because the idea is to grow organic food, as a mandatory part of healthy lifestyle, which is gaining more importance world-wide.

\section{CONCLUSION}

Technology can be used to facilitate and improve working conditions of agricultural producers. Two problems, the inability to distinguish fruits from leaves and performing tasks for only one type of fruit were successfully solved with MultiHBot, as part of fully automated greenhouse, and human interventions. Future research of the author will be focused on higher level of automation of MultiHBot. 


\section{REFERENCES}

[1] FAO 2009, The State of Food Insecurity in the World - Economic crises and lessons learned.

[2] Ball, D., Ross, P., English, A., Patten, T., Upcroft, B., Fitch, R., Sukkarieh, S., Wyeth, G., Corke, P. (n.d.) Robotics for Sustainable Broad-Acre Agriculture.

[3] Isaacs, GW 1986, 'Robotic applications in agriculture', Acta Horticulturae, no. 187, pp. 123-128.

[4] Alexandrou, L, Pelagia, S \& Pitiris, D 2006, Agricultural Statistics p. 155.

[5] Murakami, N, Ito, A, Will, JD, Steffen, M, Inoue, K, Kita, K \& Miyaura, S 2008, 'Development of a teleoperation system for agricultural vehicles', Computers and Electronics in Agriculture, vol. 63, no. 1, pp. 81-88.

[6] Murphy, RR. (2004) Human-robot interaction in rescue robotics', Systems, Man, and Cybernetics, Part C: Applications and Reviews, IEEE Transactions on, vol. 34, no. 2, pp. 138-153.

[7] Adamides, G., Berenstein, R., Ben-Halevi, I., Hadzilacos, T., Edan, Y. (n.d.). User Interface Design Principles for Robotics in Agriculture: The Case of Telerobotic Navigation and Target Selection for Spraying

[8] Clarkson, E \& Arkin, RC 2007, 'Applying Heuristic Evaluation to Human-Robot Interaction Systems'.

[9] Goodrich, MA \& Schultz, AC 2007, 'Human-robot interaction: a survey', Found. Trends Hum.-Comput. Interact., vol. 1, no. 3, pp. 203-275.

[10] Pedersen, S, Fountas, S, Have, H \& Blackmore, B 2006, 'Agricultural robots-system analysis and economic feasibility', Precision Agriculture, vol. 7, no. 4, pp. 295-308.

[11] Wang, C, Wang, F, Chen, L \& Zhang, C 2009, 'A System Design for the Testing Platform of Robot Teleoperation with Enhanced Reality Based on Binocular Vision', in Information Technology and Applications, 2009. IFITA '09. International Forum on, pp. 565-569.

[12] Roberto, O, Chen, Z, Bobby, B \& Julie, AA 2003, Interface Evaluation for Mobile Robot Teleoperation

[13] Fong, T, Thorpe, C \& Baur, C 2001, 'Advanced Interfaces for Vehicle Teleoperation: Collaborative Control, Sensor Fusion Displays, and Remote Driving Tools', Autonomous Robots, vol. 11, pp. 77-85.

[14] Bechar, A \& Edan, Y 2003, 'Human-robot collaboration for improved target recognition of agricultural robots', Industrial Robot: An international journal, vol. 30, no. 5, pp. 432436.

[15] Berenstein, R, Shahar, OB, Shapiro, A \& Edan, Y 2010, 'Grape clusters and foliage detection algorithms for autonomous selective vineyard sprayer', Intelligent Service Robotics, vol. 3, no. 4, pp. 233-243.

[16] Bangert, W., Kielhorn, A., Rahe, F., Albert, A., Biber, P., Grzonka, S., Haug, S., Michaels, A., Mentrup, D., Hänsel, M., Kinski, D., Möller, K., Ruckelshausen, A., Scholz, C., Sellmann, F., Strothmann, W., Trautz, D. (n.d.) Field-Robot-Based Agriculture: "RemoteFarming.1" and "BoniRob-Apps"

[17] Mandow, J. M. Gómez-de-Gabriel, J. L.Martínez, V. F. Muñoz, A. Ollero and A. GarcíaCerezo "The Autonomous Mobile Robot AURORA for Greenhouse Operation" Robotics and Automation Vol. 3, N4, pp. 18-28, December 1996.

[18] Acaccia, G.M., Michelini, R.C., Molfino, R.M., Razzoli R.P. (2003) Mobile robots in greenhouse cultivation: inspection and treatment of plants

[19] Sammons, P. J., Furukawa, T. Bulgin, A. (2005) Autonomous Pesticide Spraying Robot for use in a Greenhouse 
[20] Gonzalez, R., Rodriguez, F., Sanchez-Hermosilla, J., Donaire, J. G. (2009) Navigation techniques for mobile Robots in greenhouses. Applied Engineering in Agriculture. Vol. 25(2): 153-165

[21] Kitamura, S., Oka, K. (2005) Recognition and Cutting System of Sweet Pepper for Picking Robot in Greenhouse Horticulture. International Conference on Mechatronics \& Automation Niagara Falls, Canada, July 2005 